\title{
Auswirkungen von Studienkosten auf herkunftsspezifische Ungleichheiten bei der Studienaufnahme und der Studienfachwahl
}

\author{
Carina Engelhardt $\mathbb{D} \cdot$ Markus Lörz
}

Eingegangen: 9. November 2020 / Angenommen: 23. Juni 2021 / Online publiziert: 2. August 2021

(C) Der/die Autor(en) 2021

Zusammenfassung Trotz Bildungsexpansion und verschiedener bildungspolitischer Bemühungen bestehen beim Übergang ins Studium und in der Studienfachwahl bis heute erhebliche herkunftsspezifische Unterschiede. Inwieweit bildungspolitische Reformen die sozialen Ungleichheiten im Hochschulbereich verändern und welche Studierendengruppen auf solche reagieren, wurde bislang jedoch selten untersucht. Der vorliegende Beitrag beschäftigt sich daher am Beispiel der BAföG-Reform 1983 mit der Frage, inwieweit kostenbezogene Veränderungen den Übergang ins Studium und im Speziellen die Studienfachwahl beeinflussen. Ausgehend von kulturellen Reproduktionsprozessen und rationalen Entscheidungsprozessen werden verschiedene theoretische Erklärungsansätze skizziert und im Rahmen eines Differenz-inDifferenzen(DID)- und Regressions-Diskontinuitäts(RD)-Designs hinsichtlich ihrer empirischen Evidenz getestet. Die Ergebnisse zeigen, dass die BAföG-Reformen im Zeitraum von 1976-1986 zu einer Verstärkung der Ungleichheiten beim Übergang ins Studium geführt haben, aber bei der Studienfachwahl in die umgekehrte Richtung wirken. Aufgrund der Erhöhung der Studienkosten verzichten Studienberechtigte aus weniger privilegierten Familien zwar häufiger auf ein Studium, aber diejenigen, die sich für eine Studienaufnahme entscheiden, wählen in zunehmendem

\footnotetext{
Online-Anhang: www.kzfss.uni-koeln.de/sites/kzfss/pdf/Engelhardt_Lörz.pdf

C. Engelhardt $(\bowtie)$

Hochschule Weserbergland

Am Stockhof 2, 31785 Hameln, Deutschland

E-Mail: engelhardt@hsw-hameln.de

M. Lörz

Fachgruppe Soziologie, Otto-Friedrich-Universität Bamberg

Feldkirchenstr. 21, 96045 Bamberg, Deutschland

E-Mail: loerz@dzhw.eu

Bildungsverläufe und Beschäftigung, Deutsches Zentrum für Hochschul- und Wissenschaftsforschung Lange Laube 12, 30159 Hannover, Deutschland
} 
Maße lukrativere Studienrichtungen. Das Bildungsverhalten der Studienberechtigten aus privilegiertem Elternhaus wird dagegen erwartungsgemäß von der BAföGReform weniger stark beeinflusst.

Schlüsselwörter Soziale Ungleichheit · Übergang ins Studium • Bildungsentscheidung · BAföG-Reform · Hochschulbildung

\title{
Impact of Study Costs on Social Inequalities in Transition to Higher Education and Field of Study Choice?
}

\begin{abstract}
Despite educational expansion and different educational reforms, we still observe social inequalities in the transition to higher education and in the choice of field of study. There is little literature investigating to what extent education policies can change these inequalities and who is primarily affected by this. In this paper, we evaluate the impact of cost-related changes on family background-specific inequalities in the transition to higher education and the choice of field of study. We use quasi-experimental data from a 1983 German educational reform and evaluate them by using difference-in-differences estimates and a regression discontinuity design. We show that increased study costs lead less privileged people with university or other higher-education entrance qualifications to abstain more often from further higher education. For those who do decide to continue their education, however, increased study costs reduce family background-specific inequalities in the field of study. All in all, our results are in line with the theory of cultural transmission and rational choice theory.
\end{abstract}

Keywords Social inequality · Transition to higher education · Educational decision · BAföG reform · Tertiary education

\section{Einleitung}

Die empirische Bildungsforschung macht seit Jahrzehnten darauf aufmerksam, dass in Deutschland der Weg zu einem Hochschulabschluss in hohem Maße von den kulturellen und sozioökonomischen Rahmenbedingungen im Elternhaus abhängig ist (Maaz 2006; Becker und Hecken 2008; Neugebauer und Schindler 2012). Die soziale Herkunft der Schülerinnen und Schüler wirkt sich dabei nicht nur auf den Zugang und den erfolgreichen Abschluss eines Studiums aus (vertikale Ungleichheiten), sondern auch auf die Art der Bildungsbeteiligung (horizontale Ungleichheiten). Kinder aus weniger privilegierten Familien wählen weniger prestigeträchtige Studienfächer (Becker et al. 2010; Reimer und Pollak 2010), studieren häufiger an Fachhochschulen (Schindler und Reimer 2010; Lörz 2013), sind an renommierten Hochschulen unterrepräsentiert (Weiss et al. 2015; Wagner 2018) und studieren im Laufe des Studiums seltener im Ausland (Netz und Finger 2016; Lörz et al. 2016). Die Herkunftsunterschiede zeigen sich folglich nicht nur beim Zugang zu höherer Bildung, sondern auch in der Art der Bildungsbeteiligung und den extracurricularen Erfahrungen, die neben den formalen Inhalten des Bildungsweges gesammelt 
werden. Die Forschung zu sozialer Stratifizierung und sozialer Ungleichheit richtet aus diesem Grunde zunehmend den Fokus auf die feineren Unterschiede in der Bildungsbeteiligung. Insbesondere die unterschiedliche Studienfachwahl beschäftigt die Ungleichheitsforschung seit mehreren Jahrzehnten, da sich aus einer solchen die später erzielten Einkommen (Leuze und Strauß 2009; Reimer und Steinmetz 2009) und die weiterführenden Karriereoptionen (Iannelli et al. 2018) ergeben.

Auch tauchen auf der bildungspolitischen Agenda in regelmäßigen Abständen die Schwerpunkte „Abbau von Bildungsungleichheiten“ und die „Förderung von Bildungspotenzialen“ auf. Im Hochschulbereich wurde zudem in den vergangenen Jahrzehnten eine Reihe an Reformen verabschiedet, die sich sowohl auf die institutionelle Ausgestaltung (Bologna-Reform, Exzellenzinitiative) als auch auf die Finanzierung (BAföG, Studiengebühren) des Studiums beziehen. Aktuell werden vor dem Hintergrund der Coronapandemie die KfW-Studienkredite zinsfrei vergeben und im Rahmen der Überbrückungshilfe in pandemiebedingten Notlagen den Studierenden zusätzliche Finanzierungsmöglichkeiten des Studiums angeboten. Zudem wird vom Deutschen Studentenwerk bereits seit Längerem die Ausdehnung des BAföG um ein Semester und die Abschaffung der Altersgrenze gefordert (DSW 2019) - insbesondere vor dem Hintergrund dessen, dass der Anteil an BAföG-Empfängerinnen und -empfänger ${ }^{1}$ in den vergangenen Jahren sukzessive zurückgegangen ist, während der Anteil an Studienanfängern weiter steigt (Statistisches Bundesamt 2017). Die Konsequenzen solcher Reformen sind bislang allerdings weniger gut erforscht. Zwar liegen mit Blick auf den Zugang zum Studium bereits erste Erkenntnisse vor, inwieweit Bildungsreformen die Entscheidung zu studieren beeinflussen (Helbig et al. 2012; Quast et al. 2012; Neugebauer et al. 2016), allerdings wurden die Konsequenzen solcher Reformen für die feineren Unterschiede in der Art der Bildungsbeteiligung bislang noch nicht hinreichend betrachtet.

Die Auswirkungen von Bildungsreformen zu untersuchen, ist methodisch oftmals nicht ganz einfach, da es sowohl vor als auch nach der Reform einer hinreichend validen und vergleichbaren Datenbasis bedarf. Ein solcher Fall liegt mit der Reform des BAföG im Jahr 1983 und den in diesem Zeitraum regelmäßig stattfindenden flächendeckenden Studierendenbefragungen vor. Daher setzt der vorliegende Beitrag an dieser Stelle an und untersucht, inwiefern sich die Umstellung des BAföG auf ein Volldarlehen (im Jahr 1983) auf das Bildungsverhalten der verschiedenen Bildungsherkunftsgruppen ausgewirkt hat. Es handelt sich hierbei zwar um vergleichsweise alte Daten und ein historisch weit zurückliegendes Ereignis, aber anhand dieser Erkenntnisse lässt sich erkennen, inwieweit die sozialen Ungleichheiten beim Übergang ins Studium (i) und in der Studienfachwahl (ii) durch eine Erhöhung der Studienkosten zunehmen oder vielleicht auch abnehmen. Das übergeordnete Ziel besteht darin, zu überprüfen, ob ein Zusammenhang (Treatment-Effekt) gefunden werden kann oder nicht. Es geht demnach darum, Veränderungen im Zeitverlauf aufzuzeigen und nicht um eine möglichst vollständige Untersuchung der Determinanten der Studienfachwahl oder der Studienaufnahme. Um einen ersten Eindruck darüber zu erhalten, welche Aspekte aus Rational-Choice-Perspektive (Kostenüberlegung,

\footnotetext{
1 Im Folgenden wird das generische Maskulinum verwendet.
} 
Ertragsüberlegung, Leistung) von Belang sein könnten, fließen diese Überlegungen zum Teil auch in die Analyse ein.

Zur Beantwortung dieser Forschungsfragen wird zunächst im nachfolgenden Abschnitt der Forschungsstand skizziert und sowohl aus Rational-Choice-Perspektive als auch aus Perspektive der Theorie kultureller Reproduktion verschiedene theoretische Erwartungen hinsichtlich der Entwicklung sozialer Unterschiede abgeleitet (Abschn. 2). In Abschn. 3 werden die Daten, Variablen und die Analysestrategie vorgestellt und in Abschn. 4 folgt die empirische Analyse. Abschließend werden die empirischen Ergebnisse vor dem Hintergrund der theoretischen Erwartungen betrachtet und hinsichtlich weiterführender Forschungsbedarfe sowie bildungspolitischer Implikationen diskutiert (Abschn. 5).

\section{Forschungsstand und theoretische Überlegungen}

Die Ursachen herkunftsspezifischer Unterschiede am Übergang ins Studium und in der Studienfachwahl wurden bereits von einer Vielzahl an Studien untersucht (für Deutschland siehe beispielsweise Windolf 1992; Becker et al. 2010; Reimer und Pollak 2010; international siehe beispielsweise Davies und Guppy 1997; Van de Werfhorst et al. 2003; Barone et al. 2018). Es zeigt sich, dass Kinder aus privilegierten Familien die prestigeträchtigeren Studienfächer Medizin und Jura bevorzugen, während Kinder aus weniger privilegierten Familien häufiger ein technisches oder wirtschaftswissenschaftliches Studium aufnehmen (Spangenberg und Quast 2016). Nach Lörz (2012) ist es hierbei weniger der Berufsstatus der Eltern, sondern vielmehr das konkrete Berufsfeld der Eltern, welches zu diesem Ungleichheitsphänomen führt. Kinder aus Medizinerhaushalten entscheiden sich demnach eher für ein Medizinstudium, während beispielsweise Kinder aus Mathematikerhaushalten überproportional häufig ein Mathematikstudium bevorzugen. Die Ursachen dieser fachlichkulturellen Berufsvererbung sind weniger gut untersucht. Mit Blick auf die Forschung zu geschlechtsspezifischen Ungleichheiten in der Studienfachwahl könnte die Erklärung aber möglicherweise mit unterschiedlichen Rollenvorbildern im Elternhaus (Busch-Heizmann 2015), Schwerpunktsetzungen in der Schule (Heine et al. 2006), leistungsbezogenen Unterschieden (Lörz et al. 2011) oder mit einer unterschiedlichen Interessenausbildung zusammenhängen (Ochsenfeld 2016; Hägglund und Lörz 2020). Studien, die sich mit der zeitlichen Veränderung herkunftsspezifischer Unterschiede in der Studienfachwahl auseinandersetzen, liegen in Deutschland bislang nur vereinzelt vor. Georg (2005) kann für den Zeitraum 1985-2004 eine tendenzielle Zunahme der herkunftsspezifischen Ungleichheiten in der Studienfachwahl feststellen, während Reimer und Pollak (2010) dagegen zu dem Ergebnis kommen, dass die herkunftsspezifischen Ungleichheiten zwischen 1983 und 1999 weitgehend stabil geblieben sind. Diese Studien betrachten zwar herkunftsspezifische Unterschiede in der Studienfachwahl über einen längeren Zeitraum, beziehen in ihre Überlegungen allerdings keine besonderen Bildungsreformen ein. Da die wesentliche Ursache für herkunftsbedingte Unterschiede am Übergang ins Studium in einer unterschiedlichen finanziellen Kostenbelastung liegt (Becker und Hecken 2008; Daniel et al. 2018), ließe sich der Einfluss von Bildungsreformen auf die sozialen 
Ungleichheiten in der Studienfachwahl wahrscheinlich am ehesten am Beispiel von Reformen untersuchen, die sich auf die Finanzierung des Studiums beziehen. Die BAföG-Reform im Jahr $1983^{2}$ oder die Einführung von Studiengebühren im Jahr $2006^{3}$ stellen solche tiefgreifenden Veränderungen der finanzierungsbezogenen Studiensituation dar.

Die Forschung, die diese bildungspolitischen Reformen ins Zentrum der Analyse rückt, konzentriert sich im Hochschulbereich bisher vornehmlich auf die herkunftsspezifischen Unterschiede bei der generellen Entscheidung, ein Studium aufzunehmen. Baumgartner und Steiner (2006) kommen dabei auf Basis der Daten des Sozio-oekonomischen Panels (SOEP) zu dem Ergebnis, dass die BAföG-Reformen keine nennenswerten Auswirkungen auf den Studienzugang haben oder nach Steiner und Wrohlich (2008) nur einen kleinen Effekt zeigen und dabei die Bildungsherkunft keine Rolle spielt. Lörz und Schindler (2011) stellen demgegenüber auf Basis der Studienberechtigtenbefragungen des Deutschen Zentrums für Hochschul- und Wissenschaftsforschung (DZHW) zwischen 1980 und 1986 eine deutlich gesunkene Studierbereitschaft aller Studienberechtigten und eine Zunahme der Herkunftsunterschiede fest. Hinsichtlich der Konsequenzen der BAföG-Reform liegen demnach widersprüchliche Forschungsergebnisse vor. Ein ähnliches Bild zeigt sich in Deutschland mit Blick auf die Studien zum Thema Studiengebühren. Hinsichtlich der Einführung von Studiengebühren im Jahr 2006 kommen Quast et al. (2012) auf Basis der DZHW-Studienberechtigtendaten zu dem Ergebnis, dass mit der Erhöhung der Studienkosten die Studierbereitschaft insgesamt sinkt, dies aber nicht zu einer nennenswerten Verstärkung der sozialen Ungleichheiten geführt hat. Helbig et al. (2012) stellt demgegenüber weder eine sinkende Studierbereitschaft noch eine Verstärkung der sozialen Ungleichheiten fest. Weitere Studien von Hübner (2012) sowie Dietrich und Gerner (2012) schließen sich den Erkenntnissen von Quast et al. (2012) an, während Kroth (2015) wiederum zu dem Ergebnis gelangt, dass nicht nur die Studierbereitschaft sinkt, sondern auch die sozialen Ungleichheiten zunehmen. Die Befundlage ist demnach je nach verwendetem Datensatz, Analysemethode und betrachteten Bildungsreformen unterschiedlich. Zudem geht aus den vorliegenden Studien bislang nicht hervor, wie sich eine Erhöhung der Studienkosten in Deutschland auf die herkunftsspezifischen Unterschiede in der Studienfachwahl ausgewirkt hat.

Im internationalen Vergleich finden sich jedoch bereits einige Studien, die sich mit den Konsequenzen von Bildungsreformen auf den weiteren Studienverlauf auseinandersetzen. Rothstein und Rouse (2011) zeigen, dass sich die Umstellung von Studiendarlehen auf Stipendien auf die Wahl des Jobs der Absolventen einer Eliteuniversität in den USA signifikant auswirkt. Absolventen mit höheren Schulden haben tendenziell eher einkommensstarke Jobs gewählt. Sie konnten aber nicht ein-

\footnotetext{
2 Anfang der 1980er-Jahre wurde der bis dahin nicht zurückzuzahlende finanzielle Zuschuss nach dem Bundesausbildungsförderungsgesetz (BAföG) auf ein Volldarlehen umgestellt und Anfang der 1990erJahre wieder auf ein Halbdarlehen erhöht (Schmidt 2009).

3 Ende 2005 wurde entschieden, dass die Hochschulen Studiengebühren einführen dürfen. Ein Teil der Bundesländer führte daraufhin im Jahr 2006 flächendeckend Studiengebühren in Höhe von 0 bis $500 €$ ein. Im Zeitraum bis 2012 haben die meisten Bundesländer die Studiengebühren wieder abgeschafft.
} 
deutig identifizieren, ob sich die Reduktion der Studienkosten auch auf die Studienfachwahl auswirkt. Schmeiser et al. (2016) zeigen, dass Studierende in den USA überproportional ihren Major wechselten, nachdem sie über ihren aktuellen Schuldenstand informiert wurden. Im internationalen Vergleich zeigt sich demnach bereits, dass sich finanzierungsbezogene Reformen und Informationen auf das Studienverhalten auswirken können.

Zunächst gilt es jedoch, aus theoretischer Perspektive die Frage zu klären, warum sich eine Erhöhung der Studienkosten auf das Bildungsverhalten der verschiedenen Herkunftsgruppen überhaupt unterschiedlich auswirken sollte? Im Folgenden diskutieren wir dies anhand des Beispiels der BAföG-Reform aus dem Jahre 1983.

In Deutschland haben grundsätzlich alle Studierenden, deren Eltern ein Studium nicht (ausreichend) finanzieren zu können, die Möglichkeit, BAföG zu beantragen. Da Studierende aus weniger privilegiertem Elternhaus oftmals nicht über hinreichende Finanzierungsmöglichkeiten verfügen, ist das BAföG insbesondere für diese Studierendengruppen eine gute Möglichkeit, ein Studium zu finanzieren. Bis Anfang der 1980er-Jahre war das BAföG als Vollzuschuss konzipiert - d.h. die Studierenden mussten in diesen Jahren nichts zurückzahlen. Im Jahr 1983 wurde das BAföG allerdings auf ein Volldarlehen umgestellt. Diese Reform hatte insbesondere für die Studierenden aus weniger privilegiertem Elternhaus zur Konsequenz, dass nach dem Studium in teilweise nicht unerheblichem Umfang die BAföG-Schulden zunächst zurückgezahlt werden mussten. Die finanziellen Rahmenbedingungen der Studienberechtigten aus weniger privilegiertem Elternhaus haben sich demnach von einem Jahr auf das nächste (1983) schlagartig verschlechtert. Während vor 1983 für die Studierenden keinerlei BAföG-Schulden entstanden, musste zwischen 1983 und 1990 die gesamte BAföG-Summe zurückgezahlt werden. Mittlerweile sind die zurückzuzahlenden BAföG-Schulden auf maximal 10.000€ begrenzt (BAföG §18). Eine parallellaufende Entwicklung war in dieser Phase die steigende Bildungsbeteiligung im Sekundarschul- und Hochschulbereich. Mit Blick auf diese Veränderungen wird aus Perspektive der Theorie kultureller Reproduktion (Bourdieu 1982) oftmals vermutet, dass im Zuge der Bildungsexpansion höhere Bildung an Exklusivität verliert und für den intergenerationalen Statuserhalt feinere Differenzierungslinien wie beispielsweise die Wahl des Studienfachs oder des Studienorts - an Bedeutung gewinnen. Aus dieser Perspektive sind insbesondere Kinder aus privilegierten Familien bestrebt, über einen Hochschulabschluss oder die Wahl eines aussichtsreichen Studienfachs den beruflichen und sozialen Status ihrer Eltern zu reproduzieren. Zu einer ähnlichen Erwartung gelangt Lucas (2001, 2009) im Rahmen der EffectivelyMaintained-Inequality-Hypothese. Demnach sollten die privilegierten Bildungsherkunftsgruppen dann in eher qualitative (horizontale) Aspekte der Bildung investieren, wenn die quantitativen (vertikalen) Bildungsunterschiede abnehmen. Die Reproduktion sozialer Ungleichheiten findet diesen Überlegungen zufolge stärker über qualitative Aspekte der Bildungsbeteiligung statt, wenn dies allein über das quantitativ erreichte Bildungsniveau nicht mehr gelingt. Aus diesen Überlegungen geht zwar hervor, dass insbesondere die privilegierten Bildungsherkunftsgruppen mit der Expansion des Hochschulbereichs ihr Bildungsverhalten ändern und über die Zeit zunehmend in höhere Bildung investieren und zunehmend lukrativere Studienfächer wählen, es bleibt allerdings unklar, wie sich das Bildungsverhalten der weniger pri- 
vilegierten Studierendengruppen verändert, wenn die Kosten eines akademischen Bildungsweges erhöht werden.

Mit Blick auf die Grundannahmen der Rational-Choice-Theorie (Becker 1964; Boudon 1974) sollte eine Erhöhung der Bildungskosten generell zu einer sinkenden Nachfrage von Bildung führen. Allerdings hängt nach Erikson und Jonsson (1996) die Wahl des Bildungsweges neben den Kosten auch von den zu erwartenden Erträgen und Erfolgsaussichten ab. Denn solange die relativen Erträge die relativen Kosten überwiegen, ist nicht unbedingt eine Veränderung des Bildungsverhaltens zu erwarten. Eine Erhöhung der Studienkosten sollte demnach insbesondere die Studienberechtigten von einer Studienaufnahme abschrecken, die stärker kostensensibel reagieren (i), die sich von einem Studium keine allzu hohen Erträge versprechen (ii) und deren Erfolgsaussichten nicht so hoch ausfallen (iii).

Mit Blick auf die BAföG-Reform wäre nun davon auszugehen, dass insbesondere die weniger privilegierten Bildungsherkunftsgruppen kostensensibel reagieren und in Phasen, in denen der Zuschussanteil des BAföG gesenkt wird, verstärkt auf eine Studienaufnahme verzichten. Zum einen, da insbesondere einkommensschwächere Gruppen antragsberechtigt sind und eine BAföG-Reform für diese Gruppen einen höheren finanziellen Einschnitt bedeutet. Zum anderen, da für diese Herkunftsgruppen die Finanzierung des Studiums aufgrund der geringeren elterlichen Unterstützungsmöglichkeiten eine höhere Belastung darstellt. Mit der im Jahr 1983 reduzierten BAföG-Unterstützung sollte folglich insbesondere die Studierbereitschaft der weniger privilegierten Bildungsherkunftsgruppen sinken (H1).

Hinsichtlich der monetären Erträge von Bildung wäre zunächst davon auszugehen, dass diese für alle Herkunftsgruppen ähnlich ausfallen. Allerdings dürfte in Studienrichtungen, in denen die späteren Einkommen nicht ganz so hoch ausfallen (beispielsweise Soziale Arbeit, Kulturwissenschaft, Sozialwissenschaft) zu erwarten sein, dass sich dort die Wirkung der BAföG-Reform in besonderem Maße zeigt. Bei einer Erhöhung der Studienkosten sollte insbesondere die Nachfrage nach diesen Studienrichtungen sinken. In den Studienrichtungen, in denen höhere Einkommen zu erwarten sind (beispielsweise Wirtschaftswissenschaften, Medizin, Wirtschaftsingenieurwesen u.a.m.), wäre dagegen zu erwarten, dass sich die BAföG-Reform in geringerem Maße auf das Bildungsverhalten der Studierenden auswirkt oder sogar zu einer verstärkten Nachfrage dieser Studienrichtungen führt. Da sich die BAföGReform insbesondere auf die Studiensituation der weniger privilegierten Gruppen auswirkt, wäre zu erwarten, dass diese mit der BAföG-Reform seltener zur Aufnahme einer weniger lukrativen Studienrichtungen neigen - entweder, weil sie sich zugunsten eines lukrativeren Studienfachs entschieden haben oder generell auf eine Studienaufnahme verzichten (H2). Für die privilegierten Herkunftsgruppen sollte die BAföG-Reform in dieser Hinsicht keine nennenswerten Veränderungen in der Studienfachwahl hervorrufen.

Insgesamt machen die theoretischen Überlegungen deutlich, dass die BAföGReform insbesondere das Bildungsverhalten der weniger privilegierten Gruppen beeinflussen sollte. Zum einen hinsichtlich der generellen Bereitschaft zu studieren (Kostenüberlegungen), zum anderen hinsichtlich der gewählten Studienrichtung (Ertragsüberlegungen). 


\section{Daten und Methoden}

\subsection{Daten}

Zur Überprüfung der theoretischen Überlegungen ziehen wir die Studienberechtigtenbefragungen der Abschlusskohorten 1976, 1978, 1980, 1983 und 1986 heran. $^{4}$ Mit diesen Daten lässt sich die generelle Studienentscheidung sowie die spezifische Studienfachwahl der studienberechtigten Schülerinnen und Schüler vor und nach der BAföG-Reform im Jahr 1983 hinreichend betrachten. Hierbei handelt es sich um für Westdeutschland repräsentative Paneluntersuchungen des Deutschen Zentrums für Hochschul- und Wissenschaftsforschung (DZHW) zu den nachschulischen Werdegängen von Studienberechtigten, die zwischen 1976 und 1986 die Hochschulreife erworben haben. Diese Schülerinnen und Schüler wurden ein halbes Jahr nach Schulabgang (1. Welle) sowie zweieinhalb Jahre nach Schulabgang (2. Welle) über ihre Pläne, Motive sowie bisherigen und zukünftigen Werdegänge schriftlich befragt. Der Großteil der Studienberechtigten hat bis zu diesem Zeitpunkt ein Studium aufgenommen (Spangenberg und Quast 2016). Um systematische Verzerrungen zu vermeiden, werden die Daten anhand bekannter Strukturmerkmale der amtlichen Statistik gewichtet (Bundesland, Art der Schule, Art der Hochschulreife und Geschlecht) und die Ausfallprozesse zwischen der ersten und zweiten Erhebungswelle in die Berechnung der Gewichtungsfaktoren einbezogen.

Über alle Jahrgänge hinweg stand in der ersten Welle ein Sample von 88.872 Studienberechtigten zur Verfügung - dies entspricht einer Rücklaufquote von 31-33\%. Aufgrund von Panelmortalität reduziert sich das Sample in der zweiten Welle auf 64.231 Schüler. Auf Basis dieser Studienberechtigten bilden wir einen Episodendatensatz mit mehreren Beobachtungen pro Person. Dadurch ist es möglich, etwaige Fachwechsel zu berücksichtigen und die Analyse ist nicht auf die Studienfachwahl zu einem bestimmten Zeitpunkt beschränkt. Nach Ausschluss von Fällen mit fehlenden Werten in den zentralen Variablen ${ }^{5}$ verbleiben im Analysesample über alle Jahrgänge zusammengenommen 63.619 Fälle (239.530 Episoden). Bei der Analyse der Studienfachwahl reduziert sich das Analysesample über alle Jahrgänge zusammengenommen auf 39.745 Fälle (114.998 Episoden), da nur die Personen in die Analyse einbezogen werden, die ein Studium aufgenommen haben.

\subsection{Variablen}

Die zentrale abhängige Variable stellt im ersten Teil der Analyse die Entscheidung, ein Studium aufzunehmen $\left(\mathrm{Y}_{\mathrm{S}}=1\right)$ oder nicht $\left(\mathrm{Y}_{\mathrm{S}}=0\right)$ dar. Im zweiten Teil der Analyse rückt die spezifische Studienfachwahl ins Zentrum der Analyse. Hierbei werden 15 Studienrichtungen unterschieden, welche wir auf Basis der Informationen des Mikrozensus (Glockner und Storck 2012) aufsteigend nach dem erwarteten Erwerbseinkommen sortiert haben. Studienrichtungen mit einem höheren Erwerbseinkommen

\footnotetext{
${ }^{4}$ Wir betrachten nur die Jahrgänge bis 1986, da die BAföG-Reform 1990 wieder teilweise zurückgenommen wurde.

5 Siehe Tab. 1 für eine Auflistung der zentralen Variablen.
} 
wird demnach ein höherer Wert zugeordnet (beispielsweise Medizin=15) als Studienrichtungen mit einem niedrigen Erwerbseinkommen (beispielsweise Sozialwissenschaften $=11)$. Hieraus ergibt sich folgende Rangfolge: $\left(Y_{F}=1\right)$ Agrar-, Ernährungsund Forstwissenschaft, $\left(Y_{F}=2\right)$ Erziehungswissenschaften und Sport, $\left(Y_{F}=3\right)$ Kunst und Gestaltung, $\left(Y_{F}=4\right)$ Kultur- und Sprachwissenschaft, $\left(Y_{F}=5\right)$ Sozialwesen und -wissenschaft, $\left(Y_{F}=6\right)$ Psychologie, $\left(Y_{F}=7\right)$ Architektur und Bauingenieurwesen, $\left(Y_{F}=8\right)$ Wirtschaftswissenschaft, $\left(Y_{F}=9\right)$ Physik und Geowissenschaft, $\left(Y_{F}=10\right)$ Biologie, Chemie und Pharmazie, $\left(Y_{F}=11\right)$ Mathematik und Informatik, $\left(Y_{F}=12\right)$ Elektrotechnik, $\left(\mathrm{Y}_{\mathrm{F}}=13\right)$ Maschinenbau, $\left(\mathrm{Y}_{\mathrm{F}}=14\right)$ Jura und $\left(\mathrm{Y}_{\mathrm{F}}=15\right)$ Medizin.

Bei der Studienfachwahl handelt es sich demnach um eine kategoriale Variable, in der nur die Rangfolge der Studienfächer abgebildet wird. Etwaige Einkommensabstände oder Einkommensunterschiede aufgrund eines Fachhochschul- oder Universitätsstudiums können hier nicht berücksichtig werden. Das Zurückgreifen auf diese Approximation ist notwendig, da die Daten zu (durchschnittlichen) Erwerbseinkommen je Studienfach für den betrachteten Zeitraum nicht vorliegen. ${ }^{6}$ Die Rangfolge basiert auf Glockner und Storck (2012), die sich auf den Anfang der 2000er-Jahre beziehen. Seitdem ist die Rangfolge der Studienfächer nahezu unverändert geblieben, wie diverse Gehaltsreporte bis 2021 bestätigen. Das Einkommenspotenzial der verschiedenen Fachrichtungen scheint offenbar ziemlich konstant zu sein. Aufgrund der groben Aufteilung in Studienfächer - statt beispielsweise in konkrete Berufe und Branchen - ist das aber auch nicht unbedingt überraschend und es gibt wenig Grund zu der Annahme, dass sich die Einkommensrangfolge dieser Fachrichtungen nennenswert verändert hat.

Aus Tab. 1 wird ersichtlich, wie sich die Studierenden der Jahrgänge 1976, 1978, 1980, 1983 und 1986 auf die verschiedenen Studienrichtungen verteilen und inwieweit sich diese Verteilung zwischen 1980 und 1983 verändert hat. Im oberen Teil der Tabelle befinden sich die Studienrichtungen, die mit einem überdurchschnittlich hohen Einkommen verbunden sind, im unteren Teil der Tabelle finden sich die Studienrichtungen mit einem vergleichsweise niedrigen Einkommen. In der letzten Spalte wird zudem dargestellt, welche Studienfachbereiche über die Zeit prozentual zugenommen und welche abgenommen haben.

Wie angenommen, steigt nach der BAföG-Reform im Durchschnitt der Anteil der Studierenden in den Studienrichtungen, in denen höhere Einkommen zu erwarten sind (oberer Teil von Tab. 1). Die durchschnittlichen prozentualen Zuwächse in den Studienrichtungen des oberen Teils der Tabelle fallen deutlich höher aus, während die Studienrichtungen des unteren Teils der Tabelle im Durchschnitt über die Zeit von weniger Studierenden gewählt werden. Zu erkennen ist dies auch anhand des Medians (50\%), welcher 1976 noch im Bereich Architektur/Bauingenieurwesen lag und zunehmend in den einkommensstärkeren Bereich Wirtschaftswissenschaften fällt. Ein differenzierter Blick auf die einzelnen Studienrichtungen macht deutlich, dass insbesondere in der Phase zwischen 1980 und 1983 in den Wirtschaftswis-

\footnotetext{
6 Übliche Quellen für Einkommensdaten wie der Mikrozensus, Allbus und das SOEP enthalten für den betrachteten Zeitraum entweder nur Angaben zu den Einkommen oder nur zur Studienfachwahl. Dies ändert sich leider erst im Laufe der späten 1990er-Jahre. Dementsprechend können die durchschnittlichen Einkommen der einzelnen Studienfächer im betrachteten Zeitraum auch nicht selbst ermittelt werden.
} 
Tab. 1 Studierende nach Studienrichtung zwischen 1976 und 1986 (in Prozent). Quelle: DZHWStudienberechtigtenbefragung 1976-1986

\begin{tabular}{|c|c|c|c|c|c|c|}
\hline & 1976 & 1978 & 1980 & 1983 & 1986 & $\begin{array}{l}\text { Differenz } \\
(86-76)\end{array}$ \\
\hline 15. Medizin & 9,67 & 9,62 & 9,22 & 13,70 & 15,12 & $+5,45$ \\
\hline 14. Jura & 5,53 & 4,91 & 5,25 & 4,32 & 3,61 & $-1,92$ \\
\hline 13. Maschinenbau & 6,15 & 6,75 & 8,30 & 6,15 & 5,99 & $-0,16$ \\
\hline 12. Elektrotechnik & 1,35 & 1,64 & 1,32 & 1,23 & 1,30 & $-0,05$ \\
\hline 11. Mathematik/Informatik & 4,84 & 5,83 & 5,49 & 3,09 & 3,59 & $-1,25$ \\
\hline $\begin{array}{l}\text { 10. Biologie/Chemiel } \\
\text { Pharmazie }\end{array}$ & 6,77 & 7,98 & 8,46 & 6,97 & 7,02 & $+0,25$ \\
\hline 9. Physik/Geowissenschaft & 6,87 & 6,01 & 6,20 & 6,96 & 7,67 & $+0,80$ \\
\hline 8. Wirtschaftswissenschaft & 7,68 & 7,96 & 8,42 & 12,10 & 12,46 & $+4,78$ \\
\hline $\begin{array}{l}\text { 7. Architektur/ } \\
\text { Bauingenieurwesen }\end{array}$ & 3,98 & 4,35 & 3,76 & 3,28 & 3,37 & $-0,61$ \\
\hline 6. Psychologie & 18,92 & 16,88 & 15,27 & 11,78 & 10,78 & $-8,14$ \\
\hline 5. Sozialwissenschaft & 5,71 & 4,69 & 6,28 & 9,26 & 8,77 & $+3,06$ \\
\hline 4. Kultur-/Sprachwissenschaft & 7,99 & 8,82 & 8,15 & 7,45 & 7,83 & $-0,16$ \\
\hline 3. Kunst/Gestaltung & 4,58 & 4,67 & 4,64 & 4,86 & 5,70 & $+1,12$ \\
\hline $\begin{array}{l}\text { 2. Erziehungswissenschaft/ } \\
\text { Sport }\end{array}$ & 6,41 & 5,48 & 5,58 & 6,05 & 3,96 & $-2,45$ \\
\hline $\begin{array}{l}\text { 1. Agrar-/Ernährungs-/ } \\
\text { Forstwissenschaft }\end{array}$ & 3,54 & 4,41 & 3,66 & 2,81 & 2,82 & $-0,71$ \\
\hline Summe der Anteile & $100 \%$ & $100 \%$ & $100 \%$ & $100 \%$ & $100 \%$ & - \\
\hline Anzahl Studierende & 6379 & 7498 & 7505 & 7387 & 5072 & - \\
\hline
\end{tabular}

Anmerkung: Die Nummerierung der Studienrichtungen entspricht der jeweiligen Kategorie der Variablen Studienfachwahl, wobei ein höherer Wert ein höheres Einkommenspotenzial impliziert.

senschaften der Anteil der Studierenden von 8,4 auf 12,1\% steigt und im Medizinbereich von 9,2 auf 13,7\%. Gleichzeitig sinkt der Anteil der Studienrichtung Psychologie von 15,3 auf 11,8\%. Auffällig ist auch, dass die relativen Gewichte der Studienrichtungen Biologie, Chemie, Pharmazie, Mathematik, Informatik, Elektrotechnik und Maschinenbau ebenfalls abnehmen, obwohl es sich dabei auch mitunter um einkommensstarke Studienfächer handelt. Dies könnte möglicherweise aufgrund einer Abwägung des Erwartungsnutzens des Studiums eines solchen Fachs zusammenhängen, da die Wahrscheinlichkeit des Scheiterns in diesen Fachbereichen vergleichsweise hoch ist. Die Änderungen der Anteile der verschiedenen Studienrichtungen von 1980 auf 1983 sind statistisch signifikant (Pearson Chi-Quadrat $=300,58 ; \operatorname{Pr}=0,000)$.

Um zu überprüfen, ob die BAföG-Reform im Gesamten einen Einfluss auf die Studienfachwahl hatte, betrachten wir die durchschnittlichen Werte der Variable Studienrichtung. Abbildung 1 zeigt den Durchschnitt der Studienrichtung für jeden Absolventenjahrgang.

Die Variable Studienrichtung kann Werte zwischen 1 und 15 annehmen, wobei ein höherer Wert für ein höheres zu erwartendes Einkommen steht. Die Durchschnittswerte rangieren von 8,07 im Sommersemester 1979 (Abschlusskohorte 1978) und 8,98 im Sommersemester 1988 (Abschlusskohorte 1986). Im Folgenden betrachten 
Abb. 1 Durchschnittliche Studienfachwahl je Jahrgang. Quelle: DZHW-Studienberechtigtenbefragung 1976-1986

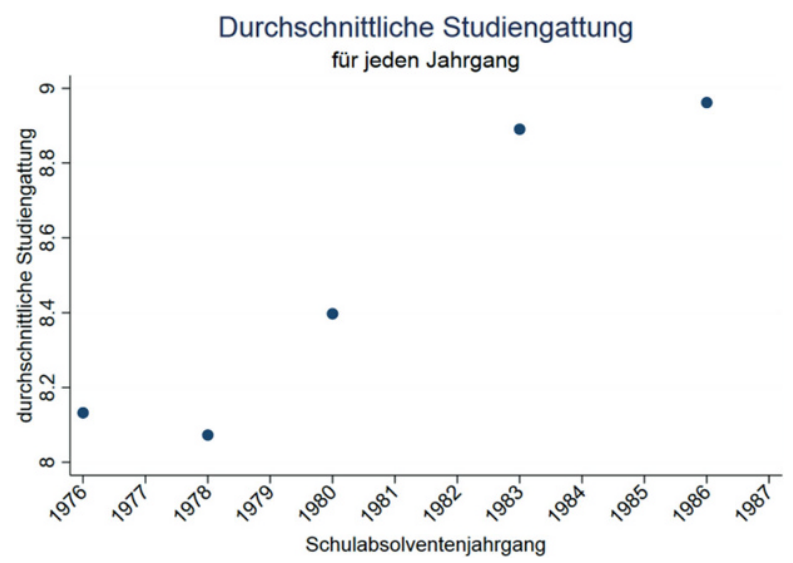

wir für jeden Absolventenjahrgang den Gesamtdurchschnitt der Studienrichtung. Für den Jahrgang 1980 beträgt dieser Wert 8,41. Die durchschnittliche Studienrichtung des Jahrgangs 1983 beträgt dagegen 8,90. Insgesamt erweckt die Abb. 1 den Eindruck, dass zum Zeitpunkt der BAföG-Reform eine sprunghafte Veränderung der Studienfachwahl zugunsten einkommensstärkerer Studienrichtungen stattgefunden hat. Diese Veränderung gilt es in den nachfolgenden Abschnitten genauer zu untersuchen.

Neben den beiden zentralen abhängigen Variablen (Studienaufnahme und Studienfachwahl) ziehen wir zur Beantwortung unserer Forschungsfragen eine Reihe weiterer Variablen heran (siehe Tab. 2).

Im Rahmen dieses Beitrags stellt die soziale Herkunft die zentrale unabhängige Variable der Analyse dar. Aus der Literatur ist zwar bekannt, dass sich die soziale Herkunft über sehr verschiedene Indikatoren messen lässt, jedoch können wir aufgrund der begrenzten Operationalisierungsmöglichkeiten in diesem Beitrag lediglich den höchsten Bildungsabschluss der Eltern verwenden: beide Elternteile ohne Hochschulabschluss $\left(\mathrm{X}_{\mathrm{H}}=0\right)$, mindestens ein Elternteil mit Hochschulabschluss $\left(\mathrm{X}_{\mathrm{H}}=1\right)$. Leider liegen in den Daten keine Informationen über das Haushaltseinkommen oder das Berufsprestige der Eltern vor. Der Bildungsabschluss der Eltern deckt zwar nur eine Facette sozialer Herkunft ab, bietet aber hinsichtlich des in diesem Beitrag

Tab. 2 Deskriptive Statistiken der zentralen Variablen. Quelle: DZHW-Studienberechtigtenbefragung 1976-1986

\begin{tabular}{|c|c|c|c|c|c|}
\hline Variable & Kodierung & Mittelwert & SD & Min & $\operatorname{Max}$ \\
\hline $\begin{array}{l}\text { Bildungs- } \\
\text { herkunft }\end{array}$ & $\begin{array}{l}\text { (1) ein Elternteil mit Hochschulabschluss; } \\
\text { (0) kein Elternteil mit Hochschulabschluss }\end{array}$ & 0,352 & 0,478 & 0 & 1 \\
\hline $\begin{array}{l}\text { Kosten- } \\
\text { überlegung }\end{array}$ & $\begin{array}{l}\text { Die baldige finanzielle Unabhängigkeit ist } \\
\text { (1) sehr bedeutend bis (6) bedeutungslos }\end{array}$ & 4,878 & 1,471 & 1 & 6 \\
\hline $\begin{array}{l}\text { Ertrags- } \\
\text { überlegung }\end{array}$ & $\begin{array}{l}\text { Einen hohen sozialen Status zu erreichen } \\
\text { ist (1) sehr bedeutend bis ( } 6 \text { ) bedeutungs- } \\
\text { los }\end{array}$ & 4,284 & 1,637 & 1 & 6 \\
\hline Leistungen & Die durchschnittliche Schulabschlussnote & 23,918 & 6,455 & 8 & 44 \\
\hline
\end{tabular}


verfolgten Analyseziels den Vorteil der Vergleichbarkeit im Kohortenverlauf. Im Folgenden sprechen wir daher von der Bildungsherkunft der Studienberechtigten.

Der Kern der Analyse lässt sich mit den drei Variablen Studienaufnahme, Studienfachwahl und Bildungsherkunft durchführen. Da neben den Konsequenzen der BAföG-Reform für die sozialen Ungleichheiten auch noch Interesse an den zugrunde liegenden Mechanismen besteht, werden in der Analyse weitere Einflussgrößen berücksichtigt. Hierbei ist es erforderlich, Variablen zu verwenden, die über die Zeit vergleichbar erhoben wurden. Um die in den Hypothesen skizzierten Überlegungen überprüfen zu können, verwenden wir einerseits die verschiedenen kosten- und ertragsbezogenen Motive (sekundäre Effekte) des nachschulischen Qualifizierungsschritts. Kostenüberlegungen werden über den Wunsch nach schneller finanzieller Unabhängigkeit gemessen und Ertragsüberlegungen über den Wunsch, beruflich Karriere zu machen. Darüber hinaus verwenden wir für die Überprüfung primärer Effekte die durchschnittliche Schulabschlussnote - auf diese Weise erhalten wir auch einen Hinweis auf die unterschiedlichen Erfolgsaussichten und deren Einfluss auf die Studienentscheidungen vor und nach der BAföG-Reform.

\subsection{Methoden}

Zur Überprüfung der theoretischen Überlegungen wird in drei Analyseschritten vorgegangen. In einem ersten Schritt wird im Rahmen eines Difference-in-Difference-Ansatzes (DID) anhand verschiedener OLS-Regressionen überprüft, ob sich die Herkunftsunterschiede beim Übergang ins Studium vor (1976-1980) und nach (1983-1986) der BAföG-Reform systematisch verändert haben. Hierbei handelt es sich um einen quasiexperimentellen Ansatz, welchem die Annahme zugrunde liegt, dass das Treatment (BAföG-Reform) nur die Treatment-Gruppe (Studierende ab 1983) und nicht die Kontrollgruppe (Studierende vor 1983) betrifft (1) und dass keine anderen Merkmale systematisch über die Zeit variieren (2). Diese Annahme könnte angesichts der steigenden Arbeitslosigkeitsquoten in dieser Phase kritisch hinterfragt werden. Zudem fällt das Arbeitslosigkeitsrisiko in geringqualifizierten Berufen meist höher aus, sodass Studierende aus Nichtakademikerfamilien vermutlich häufiger direkt mit der Arbeitslosigkeit ihrer Eltern konfrontiert sind. Allerdings sind Arbeitslosigkeitsquoten auch als Signal zu verstehen, welches alle Studierenden gleichermaßen wahrnehmen. Die BAföG-Reform wiederum betrifft nur die Studierenden, die dazu antragsberechtigt sind (Studierende aus einkommensschwächeren Familien).

In einem zweiten Schritt werden die theoretisch diskutierten Einflussgrößen schrittweise in die Analyse eingefügt und betrachtet, ob sich die über die Zeit veränderten Ungleichheitskoeffizienten unter Konstanthaltung der jeweiligen Einflussgröße erklären lassen. Auf diese Weise lassen sich empirische Hinweise für die in den Hypothesen skizzierten Überlegungen finden.

Zusätzlich werden die Ergebnisse der Studienfachwahlanalyse nochmals im Rahmen eines Regressions-Diskontinuitäts-Designs (RDD) hinsichtlich ihrer Robustheit überprüft. Auch hierbei handelt es sich um einen quasiexperimentellen Ansatz zur Identifikation von Treatment-Effekten. Der Treatment-Effekt tritt hierbei als Sprung oder Unstetigkeit der Regressionsgerade am Cutoff Point auf, wenn 
die Regressionsgerade den Zusammenhang zwischen der Zielvariablen (hier Studienfachwahl) und der Zuweisungsvariable (hier das Jahr) beschreibt. Hierbei wird überprüft, ob dieser Sprung zwischen den beiden Regressionslinien - die Niveauverschiebung - statistisch signifikant ist. Im Rahmen des RDD wird das folgende lineare Regressionsmodel ${ }^{7}$ (OLS) geschätzt:

$$
E\left(\operatorname{stgt}_{i}\right)=\beta_{0}+\beta_{1} z_{i}+\beta_{2} \widetilde{x}_{i}+\beta_{3} \widetilde{x}_{i} z_{i}+\mu_{i}
$$

wobei $\mathrm{z}_{\mathrm{i}}$ der Treatment-Indikator ist, $\beta_{1}$ den Treatment-Effekt angibt und $\widetilde{x}_{i}=$ (Jahrgang $_{i}-1983$ ) gilt, womit $\beta_{2}$ der lineare Steigungsparameter ist und $\beta_{3}$ der lineare Interaktionsparameter.

\section{Ergebnisse}

\subsection{Ungleichheitsentwicklung beim Übergang ins Studium}

In Tab. 3 werden die Ergebnisse der OLS-Regressionsanalyse hinsichtlich der Aufnahme eines Studiums (Modelle Studium: MS 1, MS 2) und mit Blick auf die Studienfachwahl (Modelle Studienfach: MF 1, MF 2) dargestellt. Es zeigt sich zum einen, dass sowohl ein Studium als auch ein lukratives Studienfach häufiger von Studienberechtigten aus akademischem Elternhaus gewählt werden. Zum anderen machen die Interaktionen mit der Zeit deutlich, dass sich diese Effekte vor und nach der BAföG-Reform systematisch unterscheiden. Mit Umstellung des BAföG steigen die herkunftsspezifischen Unterschiede in der Studienaufnahme signifikant an - insbesondere Studienberechtigte aus nichtakademischem Elternhaus verzichten nach der BAföG-Umstellung signifikant häufiger auf ein Hochschulstudium

Tab. 3 Ergebnisse OLS-Schätzung: Studienaufnahme (MS) und Studienfachwahl (MF) zwischen 1976-1986. Quelle: DZHW-Studienberechtigtenbefragung 1976-1986

\begin{tabular}{lllll}
\hline & MS 1 & MS 2 & MF 1 & MF 2 \\
\hline Jahrgang & $-0,113^{* * *}$ & $-0,118^{* * *}$ & $0,581^{* * *}$ & $0,654^{* * *}$ \\
1983-1986 (versus 1976-1980) & $(0,004)$ & $(0,005)$ & $(0,054)$ & $(0,066)$ \\
Bildungsherkunft & $0,106^{* * *}$ & $0,099^{* * *}$ & $0,323 * * *$ & $0,402^{* * *}$ \\
Akademiker (versus Nichtakademiker) & $(0,004)$ & $(0,006)$ & $(0,055)$ & $(0,069)$ \\
Interaktion & - & $0,018^{*}$ & - & $-0,210+$ \\
Akademiker*Jahrgang & & $(0,009)$ & & $(0,113)$ \\
Konstante & $0,490^{* * *}$ & $0,492 * * *$ & $8,145^{* * *}$ & $8,121^{* * *}$ \\
& $(0,003)$ & $(0,003)$ & $(0,036)$ & $(0,038)$ \\
$\mathbf{R}^{2}$ & 0,021 & 0,021 & 0,006 & 0,006 \\
$\mathbf{N}$ (Episoden) & 239.530 & 239.530 & 114.998 & 114.998 \\
\hline
\end{tabular}

Anmerkung: Auf Individual-Level geclusterte OLS-Regressionsanalyse mit robusten Standardfehlern Signifikanzniveau: $+p<0,10 ;{ }^{*} p<0,05 ; * * p<0,01 ; * * * p<0,001$

\footnotetext{
7 Für die interne Validität des RDD ist es wichtig, dass kein alternativer Grund die Diskontinuität verursacht hat (1), und dass die Pre- und Postbeziehungen optimal modelliert werden (2) können (Cappelleri und Trochim 2015). Beide Annahmen können hier als erfüllt betrachtet werden.
} 
$\left(\beta_{3}=0,018, p<0,05\right)$. Dieser Befund spricht zunächst für die in H1 postulierte theoretische Erwartung hinsichtlich einer höheren Kostensensitivität weniger privilegierter Gruppen. Hinsichtlich der Studienfachwahl zeigt sich ein gegenläufiger Effekt. Mit der Umstellung des BAföG wählen Studierende aus nichtakademischem Elternhaus häufiger eine lukrative Studienrichtung $\left(\beta_{3}=0,210, p<0,10\right)$. Dieser Befund ist allerdings lediglich auf dem $10 \%$-Niveau statistisch auffällig. Die herkunftsspezifischen Unterschiede in der Studienfachwahl nehmen demnach nach der BAföGReform tendenziell eher ab, was zunächst für die theoretischen Überlegungen aus $\mathrm{H} 2$ spricht und legt die weitere Interpretation nahe, dass die Studierenden aus nichtakademischem Elternhaus unter diesen Bedingungen nur ein Studium aufnehmen, wenn entsprechend hohe monetäre Erträge zu erwarten sind. Die BestimmtheitsmaBe $\left(R^{2}\right)$ fallen - wie erwartet - gering aus. Allerdings besteht das Hauptanliegen dieser Untersuchung darin, auffällige Veränderungen durch die BAföG-Reform aufzuzeigen, ohne die Determinanten der Studienfachwahl oder Studienaufnahme in ihrer Gesamtheit zu betrachten.

\subsection{Mechanismen}

Um die im Zeitverlauf vorgefundenen veränderten Herkunftseffekte besser verstehen zu können und mögliche Mechanismen aufzuzeigen, werden die in Tab. 3 berichteten Herkunftseffekte unter Konstanthaltung verschiedener Einflussgrößen betrachtet. In Tab. 4 sind die Ergebnisse dieser OLS-Regressionen dargestellt. In MS2 und MF2 werden die Interaktionseffekte aus Bildungsherkunft und Zeit - wie bereits in Tab. 3 - ohne weitere Kontrollvariablen dargestellt. In den darauffolgenden Modellschritten werden die unterschiedlichen Kostenüberlegungen (MS3, MF3), unterschiedlichen Ertragsüberlegungen (MS4, MF4) sowie Notenunterschiede (MS5, MF5) konstant gehalten. In MS6 und MF6 finden sich dann die Interaktionseffekte unter Berücksichtigung aller drei Einflussgrößen in einem gemeinsamen Gesamtmodell. Ziel dieser Darstellung ist es, zu zeigen, welche der betrachteten Variablen am ehesten zur Erklärung des im vorangegangenen Abschnitt beschrieben veränderten Ungleichheitsphänomens beitragen.

Hierzu gilt es, den Fokus auf die Interaktionskoeffizienten ${ }^{8}$ zu legen und zu betrachten, wie sich diese verändern, wenn die jeweiligen Rahmenbedingungen konstant gehalten werden. Werden die Kostenüberlegungen im dritten Modelschritt berücksichtigt (MS 3), reduziert sich der Interaktionseffekt hinsichtlich einer Studienaufnahme von 0,018 (MS 2, $p<0,05$ ) auf 0,011 (MS 3, n.s.) am deutlichsten und fällt im Unterschied zu den darauffolgenden beiden Modellschritten insignifikant aus. Die vor und nach der BAföG-Reform verstärkten sozialen Ungleichheiten bei der Studienaufnahme dürften demnach am ehesten mit den in H1 diskutierten Kostenüberlegungen zusammenhängen. Der Interaktionseffekt bleibt zumindest unter

\footnotetext{
8 Deswegen wurde auch für die binäre Studienaufnahme eine OLS durchgeführt, da diese bei Interaktionseffekten zu robusteren Ergebnissen führt. In Tab. A2 im Online-Anhang können die Ergebnisse einer LogitSchätzung betrachtet werden. Die marginalen Effekte ändern sich - wenn überhaupt - nur minimal, die Vorzeichen ändern sich nicht und statistische Signifikanzen sind größtenteils gleichgeblieben oder haben sich verbessert.
} 


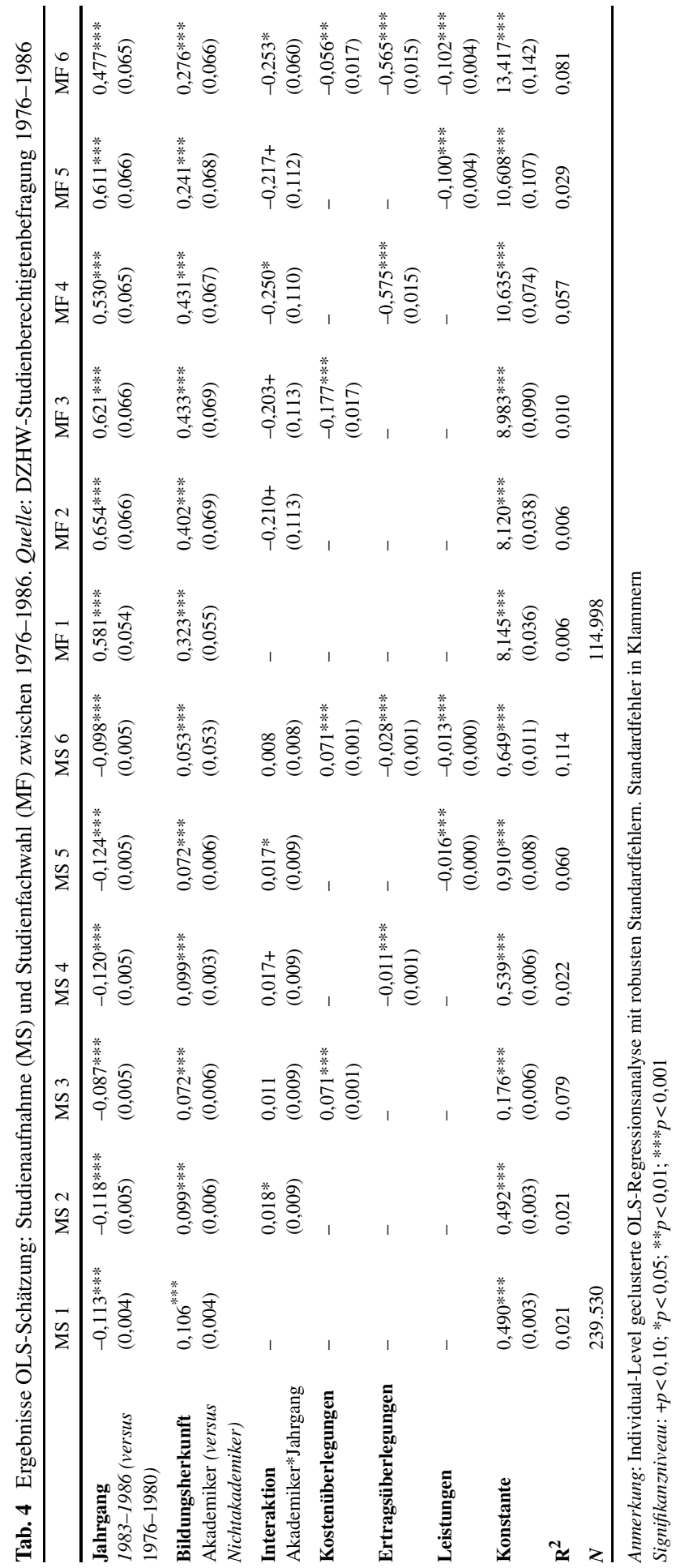


Konstanthaltung der unterschiedlichen Schulabschlussnoten und Ertragsüberlegungen weitgehend unverändert.

Mit Blick auf die veränderten sozialen Ungleichheiten in der Studienfachwahl ergibt sich ein ähnliches Bild. Werden im dritten Modellschritt die Kostenüberlegungen berücksichtigt, reduziert sich der Interaktionseffekt von $-0,210$ (MF 2, $p<0,10$ ) auf $-0,203$ (MF 3, $p<0,10$ ). Bei Berücksichtigung der Ertragsüberlegungen (MF 4) oder der Schulabschlussnoten (MF 5) zeigt sich keine Reduktion des Interaktionskoeffizienten, sondern vielmehr eine Verstärkung des Interaktionseffekts (SurpressorEffekt). Die in H2 formulierte Erwartung einer zunehmenden Wahl lukrativer Studienrichtungen resultiert demnach nicht aus einer zunehmenden Karriereorientierung der Studierenden, sondern vielmehr erneut aus einer zunehmenden Kostenorientierung der weniger privilegierten Herkunftsgruppen.

Wenngleich wir aus dieser Analyse erste Hinweise auf die zugrunde liegenden Mechanismen gewinnen, muss man einschränkend festhalten, dass es mit den einbezogenen Indikatoren nicht gelingt, das beobachtete Phänomen veränderter sozialer Ungleichheiten vollständig zu erklären. Die Verstärkung der sozialen Ungleichheiten bei der Studienaufnahme hängt zwar weitgehend mit den höheren Kostenüberlegungen der weniger privilegierten Herkunftsgruppen zusammen, aber das Phänomen abnehmender sozialer Ungleichheiten bei der Studienfachwahl lässt sich über die einbezogenen Variablen nicht erklären. Zu erkennen ist dies daran, dass im Gesamtmodell (MF 6) der Interaktionskoeffizient unter Konstanthaltung aller drei Einflussvariablen signifikant ausfällt.

\subsection{Robustness-Check}

Als Robustness-Check wird die Frage nach den Auswirkungen der BAföG-Reform zusätzlich im Rahmen eines RRD-Analysedesigns untersucht. Wie aus Abb. 2 zu erkennen ist, wählen Studienanfänger aus akademischem Elternhaus vor und nach der BAföG-Umstellung deutlich lukrativere Studienrichtungen. Allerdings zeigt sich über die Zeit ein unterschiedliches Fachwahlverhalten. Während die Studienanfänger aus akademischem Elternhaus kontinuierlich über die Zeit lukrativere Studienrichtungen wählen - was zunächst für die aus Perspektive der Theorie kulturel-
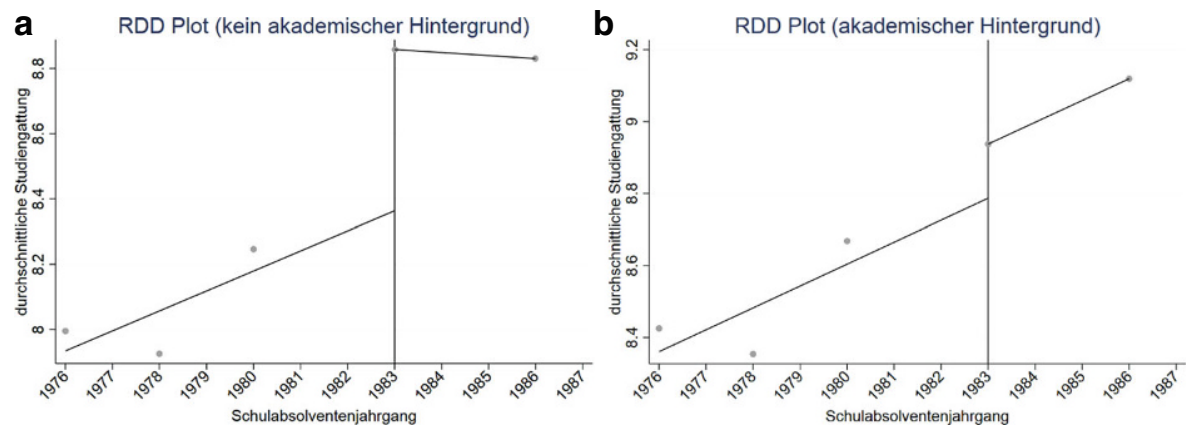

Abb. 2 Durchschnittliche Studienfachwahl nach Bildungsherkunft zwischen 1976 und 1986. a Zeigt Durchschnittswerte für Studienberechtigte ohne akademischen Hintergrund. b Zeigt ebendiese für Studienberechtigte mit akademischem Hintergrund. Quelle: DZHW-Studienberechtigtenbefragung 1976-1986 
ler Reproduktion skizzierten zunehmenden Distinkionsprozesse spricht, zeigt sich bei Studienanfängern aus nichtakademischem Elternhaus vor und nach der BAföGUmstellung eine sprunghafte Niveauverschiebung in der Lukrativität der Studienfachwahl. Zu erkennen ist dies an der vergleichsweise großen Differenz zwischen den beiden Regressionsgeraden. Zwar ist auch bei den Studienanfängern aus akademischem Elternhaus ein kleiner Bruch zu erkennen - dieser ist aber lediglich auf dem 10\%-Niveau statistisch auffällig. Der Unterschied der beiden Regressionsgeraden bei den Studienanfängern aus nichtakademischem Elternhaus fällt dagegen statistisch hoch signifikant aus (siehe Tab. A1 im Online-Anhang).

Die Ergebnisse legen demnach zwei Schlussfolgerungen nahe. Zum einen spricht die kontinuierliche Verbesserung der Studienfachwahl in der Gruppe der Studienanfänger aus akademischem Elternhaus für die von Bourdieu (1982) postulierte These des zunehmenden Strebens nach besseren Zusatzsignalen im Rahmen der Bildungsexpansion (siehe rechte Seite in Abb. 2). Der sprunghafte Anstieg der Lukrativität der Studienfachwahlen von Studienberechtigten aus nichtakademischem Elternhaus nach der BAföG-Umstellung spricht wiederum für die aus Rational-Choice-Perspektive formulierte Erwartung eines veränderten Bildungsverhaltens der weniger privilegierten Herkunftsgruppen (siehe linke Seite in Abb. 2).

\section{Diskussion}

Der vorliegende Beitrag hat sich vor dem Hintergrund der Bildungsexpansion und der BAföG-Reform im Jahr 1983 mit der Frage auseinandergesetzt, welche Konsequenz die veränderte finanzielle Studiensituation für die sozialen Ungleichheiten bei der Studienaufnahme und der Studienfachwahl hatte. Die empirischen Ergebnisse zeigen zwei gegenläufige Entwicklungen auf: Während bei der generellen Entscheidung zu studieren, die herkunftsspezifischen Unterschiede nach der BAföG-Reform signifikant zunehmen, reduzieren sich die sozialen Unterschiede tendenziell mit Blick auf die Studienfachwahl. Insbesondere Studierende aus weniger privilegiertem Elternhaus wählen nach der BAföG-Reform zunehmend einkommensstärkere Studienrichtungen. Die Ergebnisse sprechen demnach zunächst für die aus Rational-Choice-Perspektive skizzierten Überlegungen. Außerdem macht die vertiefende Analyse deutlich, dass es vermutlich insbesondere Kostenüberlegungen sind, die zu diesem veränderten Übergangsverhalten der weniger privilegierten Herkunftsgruppen beitragen. Auch für das aus Perspektive der Theorie kultureller Reproduktion skizzierte veränderte Bildungsverhalten der privilegierten Herkunftsgruppen finden sich erste Hinweise.

Die vorliegenden Daten sind für Zeitvergleiche der grundsätzlichen Unterschiede zwar sehr gut geeignet, allerdings war es mit den zu dieser Zeit (Mitte der 1970erJahre) erhobenen Daten nur eingeschränkt möglich, die zugrunde liegenden Mechanismen hinreichend herauszuarbeiten. Auch musste die Frage offengelassen werden, inwieweit sich die BAföG-Reform auf die Realisierung von Studienwünschen auswirkt und welche Konsequenzen diese Veränderungen für den weiteren Studienverlauf und Studienerfolg haben. Damit solche Fragen auch in Zukunft analysierbar sind, wird die Hochschulforschung darauf achten müssen, Daten zu sammeln, 
die über die Zeit in einer vergleichbaren Form erhoben werden und Befragungsinstrumente einzusetzen, die eine Analyse der zugrunde liegenden Prozesse besser ermöglichen.

Für die Bildungspolitik lässt sich aus unseren Ergebnissen festhalten, dass finanzierungsbezogene Reformen insbesondere das Bildungsverhalten der weniger privilegierten Herkunftsgruppen beeinflusst und dies intendierte, aber auch nichtintendierte Folgen für die sozialen Ungleichheiten am Übergang ins Studium haben kann. Der über die Jahrzehnte kontinuierlich steigende Anteil an Studierenden und der gleichzeitig sinkende Anteil an BAföG-Empfängern lässt hinsichtlich der sozialen Ungleichheit im Hochschulbereich keine gute Entwicklung erwarten. Davon sind allerdings nicht nur längerfristige Bildungsreformen betroffen, sondern auch kurzfristige Reaktionen auf aktuelle Ereignisse. Im Zuge der Corona-Pandemie (2020) haben beispielsweise viele Studierende ihre Erwerbstätigkeit verloren und auch die finanzielle Situation vieler Eltern hat sich massiv verschlechtert (Becker und Lörz 2020). Dies sind die beiden zentralen Stützen der Studienfinanzierung (Middendorff et al. 2017). Mit Blick auf unsere Ergebnisse ist nun zu vermuten, dass sich in dieser wirtschaftlich unsicheren Lage die herkunftsspezifischen Unterschiede beim Studienzugang vermutlich verstärken werden und dies auch ein verändertes Fachwahlverhalten der weniger privilegierten Gruppen hervorrufen könnte. Da überproportional die kostensensiblen Studierenden aus weniger privilegierten Gruppen existenziell auf ihre Nebenjobs angewiesen sind, benötigen insbesondere diese Studierenden alternative Finanzierungsquellen, um ihr Studium wie geplant fortführen zu können. Bildungspolitisch wurden mittlerweile als Hilfsmaßnahmen einerseits zinsfreie Studienkredite bereitgestellt. Andererseits wurde mit der „Überbrückungshilfe in pandemiebedingten Notlagen" ein Hilfspaket angeboten, welches in Form von „Stipendien“ für eine kurze Phase finanzielle Notlagen ausgleichen kann. Vor dem Hintergrund unserer Befunde wäre zu erwarten, dass Studienkredite die finanziell schwierige Situation nur unwesentlich verbessern dürften, da auch über diese Maßnahme de facto die Studienkosten insgesamt erhöht werden. Die Stipendien (Überbrückungshilfe) hingegen dürften vermutlich geeignet sein, eine weitere soziale Schieflage in der Bildungsbeteiligung zu verhindern. Allerdings werden diese Hilfsmaßnahmen bislang nur von einem kleinen Teil der Studierenden genutzt (Becker und Lörz 2020). Eine Erleichterung des Zugangs zu Unterstützungsprogrammen sowie eine Erweiterung des Adressatenkreises und eine Anpassung des Unterstützungssatzes an die gegebenen Umstände könnte gerade in Krisensituationen eine Möglichkeit darstellen, um kurzfristige finanzielle Notlagen der Studierenden zu überwinden und soziale Ungleichheiten zu vermeiden.

\footnotetext{
Danksagung Dieser Beitrag ist im Rahmen des Projekts „Studienfachwahl: Determinanten, Prozesse und soziale Ungleichheit“ des Leibniz Forschungszentrum Wissenschaft und Gesellschaft (LCSS) entstanden. Wir danken den Herausgebern und Gutachtern dieser Zeitschrift für die wertvollen Hinweise und Kommentare.
}

Funding Open Access funding enabled and organized by Projekt DEAL.

Open Access Dieser Artikel wird unter der Creative Commons Namensnennung 4.0 International Lizenz veröffentlicht, welche die Nutzung, Vervielfältigung, Bearbeitung, Verbreitung und Wiedergabe in jeglichem Medium und Format erlaubt, sofern Sie den/die ursprünglichen Autor(en) und die Quelle ord- 
nungsgemäß nennen, einen Link zur Creative Commons Lizenz beifügen und angeben, ob Änderungen vorgenommen wurden.

Die in diesem Artikel enthaltenen Bilder und sonstiges Drittmaterial unterliegen ebenfalls der genannten Creative Commons Lizenz, sofern sich aus der Abbildungslegende nichts anderes ergibt. Sofern das betreffende Material nicht unter der genannten Creative Commons Lizenz steht und die betreffende Handlung nicht nach gesetzlichen Vorschriften erlaubt ist, ist für die oben aufgeführten Weiterverwendungen des Materials die Einwilligung des jeweiligen Rechteinhabers einzuholen.

Weitere Details zur Lizenz entnehmen Sie bitte der Lizenzinformation auf http://creativecommons.org/ licenses/by/4.0/deed.de.

\section{Literatur}

Barone, Carlo, Moris Triventi und Giulia Assirelli. 2018. Explaining social inequalities in access to university. European Sociological Review 34(5):554-569.

Baumgartner, Hans Jörg, und Viktor Steiner. 2006. Does More Generous Student Aid Increase Enrolment Rates into Higher Education? Evaluating the German Student Aid Reform of 2001. IZA Discussion Paper No. 2034.

Becker, Gary Stanley. 1964. Human Capital. New York: Columbia University Press.

Becker, Karsten, und Markus Lörz. 2020. Studieren während der Corona-Pandemie: Die finanzielle Situation der Studierenden und mögliche Auswirkungen auf das Studium. DZHW Brief 09/2020.

Becker, Rolf, und Anna Etta Hecken. 2008. Warum werden Arbeiterkinder vom Studium an Universitäten abgelenkt? Zeitschrift für Soziologie 36(2):3-29.

Becker, Rolf, Sigrid Haunberger und Frank Schubert. 2010. Studienfachwahl als Spezialfall der Ausbildungsentscheidung und Berufswahl. Zeitschrift für Arbeitsmarktforschung 42(4):292-310.

Boudon, Raymond. 1974. Education, opportunity, and social inequality. New York: Wiley.

Bourdieu, Pierre. 1982. Die feinen Unterschiede. Kritik der gesellschaftlichen Urteilskraft. 1. Auflage. Frankfurt a. M.: Suhrkamp.

Busch-Heizmann, Anne. 2015. Supply-Side Explanations for Occupational Gender Segregation: Adolescents' Work Values and Gender-(A)Typical Occupational Aspirations. European Sociological Review 31:48-64.

Cappelleri, Joseph C., und William M. Trochim. 2015. Regression Discontinuity Design. In International Encyclopedia of the Social \& Behavioral Sciences, Hrsg. James D. Wright, 152-159. Elsevier.

Daniel, Annabell, Rainer Watermann und Kai Maaz. 2018. Sind studienbezogene Kosten-Nutzen-Abwägungen veränderbar? Zeitschrift für Erziehungswissenschaft 21(3):535-563.

Davies, Scott, und Neil Guppy. 1997. Fields of Study, College Selectivity, and Student Inequalities in Higher Education. Social Forces 75(4):1417-1438.

Deutsches Studentenwerk. 2019. So sieht das BAföG der Zukunft aus. Pressemitteilung vom 27.11.2019. Berlin.

Dietrich, Hans, und Hans-Dieter Gerner. 2012. The effects of tuition fees on the decision for higher education. Economics Bulletin 32:2407-2413.

Erikson, Robert, und Jan O. Jonsson. 1996. Explaining Class Inequality in Education. In Can Education be Equalized? Hrsg. Robert Erikson und Jan O. Jonsson, 1-63. Boulder, CO: Westview.

Georg, Werner. 2005. Studienfachwahl: Soziale Reproduktion oder fachkulturelle Entscheidung. ZA-Information 57.

Glockner, Daniela, und Johanna Storck. 2012. Uni, Fachhochschule oder Ausbildung - welche Fächer bringen die höchsten Löhne. DIW Wochenbericht 13:3-8.

Hägglund, Anna Erika, und Markus Lörz. 2020. Warum wählen Männer und Frauen unterschiedliche Studienfächer? Zeitschrift für Soziologie 49(1):66-86.

Heine, Christoph, Christian Kerst, Jürgen Egeln, Elisabeth Müller und Sang-Min Park. 2006. Ingenieurund Naturwissenschaften: Traumfach oder Albtraum? ZEW Wirtschaftsanalysen, Band 81. BadenBaden: Nomos.

Helbig, Marcel, Tina Baier und Anna Kroth. 2012. Die Auswirkung von Studiengebühren auf die Studierneigung in Deutschland. Zeitschrift für Soziologie 41:227-246.

Hübner, Malte. 2012. Do tuition fees affect enrollment behavior? Evidence from a 'natural experiment' in Germany. Economics of Education Review 31:949-960. 
Iannelli, Christina, Adam Gamoran und Lindsay Paterson. 2018. Fields of study: Horizontal or vertical differentiation within higher education sectors? Research in Social Stratification and Mobility 57:11-23.

Kroth, Anna. 2015. The Effects of the Introduction of Tuition on College Enrollment in Germany: Results from a Natural Experiment With Special Reference to Students from Low Parental Education Backgrounds.

Leuze, Kathrin, und Susanne Strauß. 2009. Lohnungleichheiten zwischen Akademikerinnen und Akademikern: Der Einfluss von fachlicher Spezialisierung, frauendominierten Fächern und beruflicher Segregation. Zeitschrift für Soziologie 38:262-281.

Lörz, Markus. 2012. Mechanismen sozialer Ungleichheit beim Übergang ins Studium: Prozesse der Statusund Kulturreproduktion. In Soziologische Bildungsforschung, Kölner Zeitschrift für Soziologie und Sozialpsychologie, Sonderband 52, Hrsg. Heike Solga und Rolf Becker, 302-324. Wiesbaden: VS.

Lörz, Markus. 2013. Differenzierung des Bildungssystems und soziale Ungleichheit. Zeitschrift für Soziologie 42:118-137.

Lörz, Markus, und Steffen Schindler. 2011. Bildungsexpansion und soziale Ungleichheit: Zunahme, Abnahme oder Persistenz ungleicher Chancenverhältnisse - eine Frage der Perspektive? Zeitschrift für Soziologie 40(6):458-477.

Lörz, Markus, Nicolai Netz und Heiko Quast. 2016. Why do students from underprivileged families less often intend to study abroad? Higher Education 72(2):153-174.

Lörz, Markus, Steffen Schindler und Jessica G. Walter. 2011. Gender inequalities in higher education: extent, development and mechanisms of gender differences in enrolment and field of study choice. Irish Educational Studies 30:179-198.

Lucas, Samuel R. 2001. Effectively maintained inequality: Education transitions, track mobility, and social background effects. American Journal of Sociology 106:1642-1690.

Lucas, Samuel R. 2009. Stratification Theory, Socioeconomic Background, and Educational Attainment: A Formal Analysis. Rationality and Society 21:459-511.

Maaz, Kai. 2006. Soziale Herkunft und Hochschulzugang. Effekte institutioneller Öffnung im Bildungssystem. Wiesbaden: VS.

Middendorff, Elke, Beate Apolinarski, Karsten Becker, Philipp Bornkessel, Tasso Brandt, Sonja Heißenberg und Jonas Poskowsky. 2017. Die wirtschaftliche und soziale Lage der Studierenden in Deutschland 2016.

Netz, Nicolai, und Claudia Finger. 2016. New Horizontal Inequalities in German Higher Education? Social Selectivity of Studying Abroad between 1991 and 2012. Sociology of Education 89(2):79-98.

Neugebauer, Martin, und Steffen Schindler. 2012. Early transitions and tertiary enrolment: The cumulative impact of primary and secondary effects on entering university in Germany. Acta Sociologica 55:203-205.

Neugebauer, Martin, Sebastian Neumayer und Bettina Alesi. 2016. More diversion than inclusion. Research in Social Stratification and Mobility 45:51-62.

Ochsenfeld, Fabian. 2016. Preferences, constraints, and the process of sex segregation in college majors: A choice analysis. Social Science Research 56:117-132.

Quast, Heiko, Heike Spangenberg, Bettina Hannover und Edith Braun. 2012. Determinanten der Studierbereitschaft unter besonderer Berücksichtigung von Studiengebühren. Zeitschrift für Erziehungswissenschaft 15(2):305-326.

Reimer, David, und Reinhard Pollak. 2010. Educational Expansion and Its Consequences for Vertical and Horizontal Inequalities in Access to Higher Education in West Germany. European Sociological Review 26:415-430.

Reimer, David, und Stephanie Steinmetz. 2009. Highly educated but in the wrong field? European Societies 11(5):723-746.

Rothstein, Jesse, und Cecilia Elena Rouse. 2011. Constrained after college: Student loans and early-career occupational choices. Journal of Public Economics 95(1-2):149-163.

Schindler, Steffen, und David Reimer. 2010. Soziale Ungleichheit und differenzierte Ausbildungsentscheidungen beim Übergang zur Hochschule. In Vom Kindergarten bis zur Hochschule, Hrsg. Birgit Becker und David Reimer, 251-283. Wiesbaden: VS.

Schmeiser, Maximilian, Christiana Stoddard und Carly Urban. 2016. Student loan information provision and academic choices. American Economic Review 106(5):324-28.

Schmidt, Nora. 2009. Bundesausbildungsförderung von den Anfangen bis 2007. Wirtschaft und Statistik 2(2009):157-168.

Spangenberg, Heike, und Heiko Quast. 2016. Bildungsentscheidungen und Umorientierungen im nachschulischen Verlauf. Forum Hochschule 2016 (5). Hannover: DZHW. 
Statistisches Bundesamt. 2017. Bildung und Kultur. Studierende an Hochschulen. Wintersemester 2016/2017 (Fachserie 11, Reihe 4.1).

Steiner, Viktor, und Katharina Wrohlich. 2008. Financial Student Aid and Enrollment into Higher Education. DIW Discussion Paper No. 805.

Van De Werfhorst, Herman G., Alice Sullivan und Sin Yi Cheung. 2003. Social Class, Ability and Choice of Subject in Secondary and Tertiary Education in Britain. British Educational Research Journal 29(1):41-62.

Wagner, Andreas. 2018. Fördert die Exzellenzinitiative soziale Ungleichheit bei der Hochschulwahl? Zeitschrift für empirische Hochschulforschung 2(2):133-154.

Weiss, Felix, Steffen Schindler und Maria Gerth. 2015. Hochschulrankings als Kriterium für neue soziale Ungleichheit im tertiären Bildungssystem? Zeitschrift für Soziologie 44(5):366-386.

Windolf, Paul. 1992. Fachkultur und Studienfachwahl. Ergebnisse einer Befragung von Studienanfängern. Kölner Zeitschrift für Soziologie und Sozialpsychologie 42:76-98.

Carina Engelhardt 1987, Dr. rer. pol., Lehrkraft für besondere Aufgaben an der Hochschule Weserbergland. Forschungsgebiete: Ungleichheitsforschung, Verhaltensökonomik, Methoden der empirischen Wirtschafts- und Sozialforschung. Veröffentlichungen: What Do Germans Think and Know About Income Inequality? A Survey Experiment. Socio-Economic Review (mit A. Wagner).

Markus Lörz 1976, Dr. rer. soc., Vertretung der Professur für Soziologie, insbesondere soziale Ungleichheit an der Otto-Friedrich-Universität Bamberg, Projektleitung und Verbundkoordination am Deutschen Zentrum für Hochschul- und Wissenschaftsforschung. Forschungsgebiete: Bildungs- und Arbeitsmarktforschung, Ungleichheitsforschung, Methoden der empirischen Sozialforschung und Längsschnittsdatenanalyse. Veröffentlichungen: Mechanismen sozialer Ungleichheit: Prozesse der Status- und Kulturreproduktion Kölner Zeitschrift für Soziologie und Sozialpsychologie Sonderheft 52, 2012; Gender Differences in Higher Education from a Life Course Perspektive. Higher Education 77, 2018 (mit K. Mühleck); Intersektionalität im Hochschulbereich. Zeitschrift für Erziehungswissenschaft 22, 2019. 